\title{
On Pilot-Symbol-Assisted Carrier Synchronization for DVB-S2 Systems
}

\author{
Alan Barbieri and Giulio Colavolpe, Member, IEEE
}

\begin{abstract}
We consider the problem of carrier synchronization in future 2nd-generation satellite digital video broadcasting (DVB-S2) receivers. In this scenario, this task is made harder by the complexity constraints, related to the use of consumer-grade equipment. Making use of the distributed pilot symbols of the DVB-S2 standard, low-complexity techniques for fine frequency estimation and for detection in the presence of a strong phase noise, typical of consumer-grade equipment, will be proposed. The performance of the described algorithms will be analysed in detail through computer simulations.
\end{abstract}

Index Terms-Carrier synchronization, DVB-S2 systems, iterative detection and decoding, phase noise, satellite communications.

\section{INTRODUCTION}

I N FUTURE 2nd-generation satellite digital video broadcasting (DVB-S2) systems [1], carrier synchronization is a hard task. First of all, at the very low operating signal-to-noise ratio (SNR) of some of the modulation and coding (MODCOD) formats, in particular those based on the quaternary phase shift keying (QPSK) modulation and the low-density parity-check (LDPC) codes with the lowest rates, frequency estimation is not sufficiently accurate and can be degraded by the occurrence of outliers. On the other hand, for those MODCODs working at high SNR values, namely those based on amplitude phase shift keying (APSK) signals and the highest code rates, the main problem is represented by the phase noise which is particularly strong, due to the use of consumer-grade equipment and possible low signaling rates. The phase noise also limits the accuracy of any frequency estimator for high SNR values [2]. Hence, it is particularly difficult to find a single low-complexity solution for carrier synchronization that could be adopted for all MODCODs and all signaling rates.

In this semi-tutorial paper, we report the solution designed in the context of the "Study of enhanced digital transmission techniques for broadband satellite digital transmissions (BSDT)," funded by the European Space Agency [3]. A coarse frequency synchronization is preliminary accomplished through an automatic frequency control (AFC) loop [3]. Although this block

Manuscript received July 28, 2006; revised May 18, 2007. This work is part of the "Study of enhanced digital transmission techniques for broadband satellite digital transmissions (BSDT)" supported in part by the European Space Agency, ESA-ESTEC, Noordwijk, The Netherlands, under Contract 19370. This paper was presented in part at the 9th International Workshop on Signal Processing for Space Communications (SPSC 2006), ESTEC, Noordwijk, The Netherlands, September 2006.

A. Barbieri is with the Dipartimento di Ingegneria dell'Informazione, Universita di Parma, I-43100 Parma, Italy and also with the Ming Hsieh Department of Electrical Engineering, University of Southern California, Los Angeles, CA 90089-2560 USA (e-mail: barbieri@ @lc.unipr.it).

G. Colavolpe is with the Dipartimento di Ingegneria dell'Informazione, Universita di Parma, I-43100 Parma, Italy (e-mail: giulio@unipr.it).

Color versions of one or more of the figures in this paper are available online at http://ieeexplore.ieee.org.

Digital Object Identifier 10.1109/TBC.2007.903603 is not necessary for the highest signaling rates, since the uncompensated frequency offset normalized to the symbol rate is low enough to guarantee that frame and timing synchronization can be successfully performed, for lower signaling rates it is practically unavoidable. From the design point of view, this coarse AFC loop does not represent a concern. In fact, a classical first-order loop, with an error signal generated according to the delay-and-multiply algorithm [4], is sufficient to guarantee the required performance [3]. We would like to simply mention that it is necessary to adaptively compensate the amplitude distortions on the received signal, mainly due to the low-noise block and the coaxial cable at the consumer side, since they would produce a bias in the coarse frequency estimate of the AFC loop [5]. In addition, in order to avoid an increase of the already strong phase noise, due to the phase jitter of the AFC loop, the receiver can adopt the following technique. The output of the AFC loop, at the beginning of each codeword, is used to derotate the entire codeword before the further process of fine frequency estimation and compensation and detection/decoding in the presence of phase noise, that will be described in this paper. In other words, although the AFC is still running, we use its output frozen at the beginning of each codeword. In this way, each codeword is not only affected by a constant frequency error equal to the instantaneous frequency error of the AFC loop at the beginning of the codeword, but also by the entire Doppler rate (and also by the received phase noise). However, it can be shown that the amount of this Doppler rate does not affect the performance of the algorithm we propose for joint detection and decoding in the presence of phase noise and therefore can be ignored [3].

The fine frequency estimation and the problem of detection and decoding in the presence of phase noise deserve a greater attention [6], [7] and in this paper we will focus on them. The solution we report is a merging of both new and already proposed techniques customized for this scenario-in this sense this is a semi-tutorial paper. In particular, after the description in Section II of the system model, in Section III we will consider the frequency estimation of the residual frequency offset after the coarse AFC loop. This residual frequency offset will be assumed constant over a frame and, due to the coherence time of the AFC loop, independent frame by frame [3]. The low-complexity technique that will be described makes use of distributed pilot symbols, as in the DVB-S2 standard, and the presence of the decoder. In fact, by using an autocorrelation-based estimation algorithm already proposed in the literature [4], [8], customized for the pilot symbol allocation at hand, a set of multiple estimates is identified leaving to the decoder the selection of the final estimate. The more challenging problem, that is the detection and decoding in the presence of phase noise, will be faced in Section IV. The described solution is based on an iterative algorithm already proposed by the authors in [9]. This algorithm will be briefly reviewed in this paper. Since the algorithm works with a serial schedule, which is not suited to a parallel 


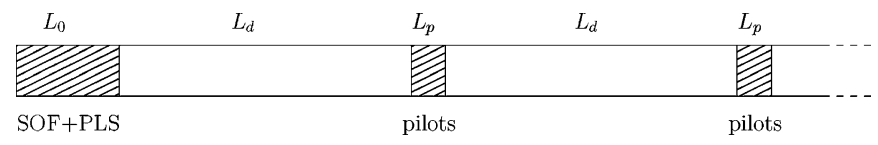

Fig. 1. Pictorial representation of a DVB-S2 frame.

implementation of the detector/decoder, and requires the perfect knowledge of the channel gain and of the operating SNR, we propose a new hybrid serial/parallel schedule and a new algorithm for the gain and SNR estimation. Finally, in Section V the performance of the described algorithms will be discussed, whereas in Section VI some conclusions will be drawn.

\section{SYSTEM MODEL}

We consider the transmission of a sequence of $K$ complex modulation symbols $\mathbf{c}=\left(c_{0}, c_{1}, \ldots, c_{K-1}\right)$ over an additive white Gaussian noise (AWGN) channel affected by an uncompensated frequency error $\nu$, after the coarse AFC loop, and a time-varying phase $\theta_{k}$. Symbols $c_{k}$ are linearly modulated. Assuming Nyquist transmitted pulses, matched filtering, and phase variations slow enough so as no intersymbol interference arises, the discrete-time baseband equivalent channel model at the receiver is given by

$$
r_{k}=A c_{k} e^{j\left(2 \pi \nu k T+\theta_{k}\right)}+w_{k}, \quad a n k=0, \ldots, K-1,
$$

being $A$ an unknown positive real gain, $T$ the symbol duration, and $\left\{w_{k}\right\}$ the additive noise samples, assumed independent and identically distributed, complex, circularly symmetric Gaussian random variables, each with mean zero and variance equal to $2 \sigma^{2}$. We assume that the sequence $\mathbf{c}$ is a codeword of the channel code $\mathcal{C}$ constructed over an $M$-ary modulation constellation $\mathcal{X} \subset \mathbb{C}$. We include the preamble and the pilot symbols (known to the receiver) as a part of the code $\mathcal{C}$.

In Fig. 1, a pictorial representation of the DVB-S2 frame format is reported. At the beginning of each frame $L_{0}=90$ known symbols, representing the start-of-frame (SOF) and the physical layer signaling (PLS) code, are inserted. Then, $P$ pilot fields, of $L_{p}=36$ symbols each, are inserted every $L_{d}=1440$ coded symbols. The number $P$ of pilot fields depends on the employed modulation (for example, $P=22$ for QPSK, while $P=8$ for 32 -APSK).

The vector of channel phases $\boldsymbol{\theta}=\left(\theta_{0}, \theta_{1}, \ldots, \theta_{K-1}\right)$ is random, unknown to both transmitter and receiver, and statistically independent of $\mathbf{c},\left\{w_{k}\right\}, \nu$, and $A$. As already mentioned, we assume that the residual frequency offset $\nu$ after the coarse $\mathrm{AFC}$ is constant over a frame and independent frame by frame. The same hypothesis will be assumed for the gain $A$. In fact, in the adaptive coding and modulation (ACM) mode of the DVB-S2 standard [1], the received signal power on codewords pertaining to different modulations can be different.

\section{Fine FreQUENCY ESTIMATION}

The aim of the fine frequency estimation block is to reduce the residual offset $\nu$ in (1) to a value tolerable by the detection algorithm. In order to find this target value, it is necessary to anticipate some concepts related to the detection algorithm that will be described in Section IV. It is a data-aided/
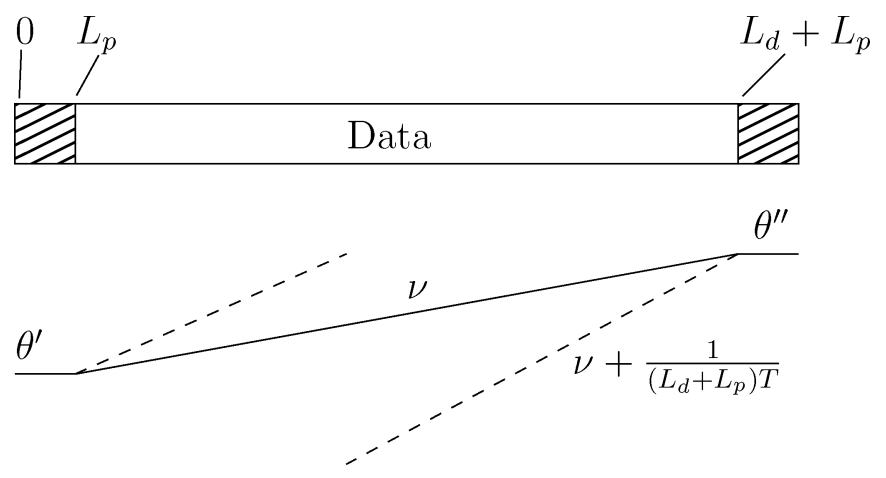

Fig. 2. Pictorial representation of a cycle slip event.

soft-decision-directed (DA/sDD) soft-input soft-output (SISO) algorithm which, at the first iteration, exploits the pilot symbols only. Let us consider Fig. 2, representing a part of a typical transmitted frame between two consecutive pilot fields. Phase variations between the two consecutive fields are mainly induced by the residual frequency offset $\nu$ and, secondarily, by the phase noise. Clearly, two values of the residual frequency offset, normalized to the signaling rate, which differ by a multiple of $1 /\left(L_{d}+L_{p}\right)$ cannot be distinguished by the detection algorithm, since they induce the same phase on each pilot field. However, they induce a completely different phase variation on data symbols between the pilot fields. Therefore, it should be clear that it is required to have a residual normalized frequency error, after the fine frequency estimation and compensation block, within the range ${ }^{1} \pm 1 / 2\left(L_{d}+L_{p}\right)$. If this is not the case, the phase estimates on data symbols between two pilot fields are completely wrong and the frame cannot be decoded, resulting in a high number of bit errors. This event was already described in [10], where the authors called it cycle slip, although it should not be confused with the cycle slip in a phase-locked loop.

Hence, the aim of the fine frequency estimation algorithm is to reduce the frequency offset such that $P\left\{|\nu T|>1 /\left(2\left(L_{d}+\right.\right.\right.$ $\left.\left.\left.L_{p}\right)\right)\right\}<F E R_{\text {target }}$, where $F E R_{\text {target }}$ is the target frame-error rate (FER). Indeed, if $\nu$ is inside the required range, it will be perfectly compensated by the detection algorithm. Under the hypothesis of a zero-mean Gaussian-distributed residual frequency error after the fine frequency estimation and compensation block, the above mentioned requirement is equivalent to require a root mean square error (RMSE) less than about $1 / 6$ of the maximum tolerable value. Hence, a very accurate frequency estimate, with a RMSE in the order of $10^{-5}$, is necessary. A pure DA frequency estimation algorithm cannot give the required accuracy [3]. On the other hand, a mixed data-aided/ non-data-aided (DA/NDA) estimation algorithm could, in principle, work well. We considered the following technique. A DA algorithm is carried out over the preamble (of size $L_{0}$ ), by using for example the Mengali and Morelli (MM) algorithm [4], [8], thus obtaining an estimate $\hat{\nu}_{\mathrm{DA}}$. The resulting estimate is affected by a quite large residual error, due to the limited preamble size. Then, a NDA search is carried out in a limited range around $\hat{\nu}_{\mathrm{DA}}$ by means of the Rife and Boorstyn (RB) algorithm [4], [11], which is the only algorithm allowing local searches,

\footnotetext{
${ }^{1}$ For the standard DVB-S2 pilot symbol distribution, since $L_{d}=1440$ and $L_{p}=36$, the range becomes $\pm 3.38 \cdot 10^{-4}$.
} 
working on $L_{\mathrm{NDA}}$ symbols, with $L_{\mathrm{NDA}} \gg L_{0}^{2}$. The new estimate $\hat{\nu}_{\mathrm{NDA}}$ is used as the final estimate.

The algorithm just described is motivated by the fact that a DA estimator over the preamble is characterized by a bad accuracy, but with a much lower outlier probability with respect to the NDA search, which on the other hand has a better accuracy since it uses a larger number of symbols. With the proposed approach, the probability of occurrence of outliers is dominated by the DA step, whereas the accuracy depends only on the number of symbols employed in the NDA search. Theoretically, this solution could reach a very good performance by indefinitely increasing the NDA observation window. However, the computational complexity may become an issue in such a situation. For this reason, we propose a different approach.

As a final remark, we would like to point out that a more sparse pilot distribution would have led to less stringent constraints on the residual frequency offset, thus leading to lower complexity estimation algorithms. On the other hand, in the absence of pilot symbols the maximum tolerable frequency offset decreases dramatically. Hence an estimation algorithm characterized by an extremely smaller RMSE should be employed in this situation.

\section{A. Data-Aided and Code-Aided Estimation Algorithm}

We propose here a solution to the problem of the fine frequency estimation, with a good robustness and a low computational complexity, which takes advantage of the distributed pilot symbols and of the presence of the decoder, and whose only disadvantage is a slight increase in the decoding latency. It is based on a three-step procedure:

Coarse DA step. A DA frequency estimate $\hat{\nu}_{1}$ is first computed on the preamble, for example by using the MM algorithm [4], [8].

Fine DA step. A new DA estimate $\hat{\nu}_{2}$ is derived, based on the distributed pilot symbols, by means of a reduced-complexity MM algorithm: ${ }^{3}$

$$
\begin{array}{r}
\hat{\nu}_{2}=\frac{1}{2 \pi\left(L_{d}+L_{p}\right) T} \arg \left[\sum_{p=1}^{P-1} \sum_{k=0}^{L_{p}-1} z_{L_{0}+L_{d}+p\left(L_{d}+L_{p}\right)+k}\right. \\
\left.\cdot z_{L_{0}+L_{d}+(p-1)\left(L_{d}+L_{p}\right)+k}^{*}\right]
\end{array}
$$

where $z_{n}=r_{n} c_{n}^{*} e^{-j 2 \pi \hat{\nu}_{1} n T}$. It is worth noticing that the estimator in (2) exhibits a very low RMSE, since exploits the large distance between the pilot symbols, but also an estimation range limited in $\pm\left(1 / 2\left(L_{d}+L_{p}\right) T\right)$. Therefore, in practice a set of estimates of the form $\hat{\nu}_{1}+\hat{\nu}_{2}+\left(\ell /\left(L_{d}+L_{p}\right) T\right), \ell=0, \pm 1, \pm 2, \ldots$, are obtained. Depending on the accuracy of the first estimation step

\footnotetext{
${ }^{2}$ It is worth noting that the NDA search may be performed over non-consecutive symbols, provided that they are uniformly distributed inside the frame with a distance $D$ one of each other. It is indeed well known [12] that, when $D$ increases, the CRB decreases and, hence, the estimation accuracy improves. This is true also in the presence of phase noise [2]. Moreover, the symbols employed for the NDA search could be split into blocks, the RB algorithm carried out independently over each block, and the final likelihood function (LF) obtained by averaging the LFs related to each block.

${ }^{3}$ It can be easily verified that it is the MM algorithm using one autocorrelation term only.
}

(namely, the coarse DA), the number of valid estimates which cannot be distinguished changes, although in practice five estimates are often sufficient. Hence, a selection step is required.

Selection. A code-aided selection algorithm is carried out to choose one among the several estimates obtained in the previous steps. The employed selection algorithm is based upon a simple consideration: when the iterative joint detection and decoding algorithm described in Section IV starts with the correct frequency, the bit errors, as well as the code syndrome, fall down very quickly, provided that the SNR is above the convergence threshold. On the contrary, with the wrong frequency, the bit errors and the code syndrome remain stuck at very high values. Therefore, a straightforward way to know if the trial frequency offset value is correct or wrong, is to check the code syndrome (which is always done in the LDPC decoding) after one decoding iteration.

The approach based on multiple trial values and a code-aided selection was already proposed in [13] for phase uncertain channels and extended to the case of joint detection and decoding in the presence of phase and frequency uncertainties in [14]. However, in [13], [14] the trial values are obtained according to different criteria. As a final remark, we would like to point out that in order to further decrease the probability of the event "choice of a wrong frequency estimate", we can perform more than one iteration for each candidate frequency estimate. In fact, for the correct frequency value, under the hypothesis of convergence of the iterative detection and decoding algorithm, the syndrome value goes down to zero with the iterations, whereas for a wrong frequency it remains stuck at a very large value.

\section{The CBC ALGORITHM}

In [9], based on the framework of factor graphs (FGs) and the sum-product algorithm (SPA), the problem of iterative detection and decoding of channel codes transmitted over channels affected by phase noise has been faced. The approach is Bayesian, i.e., the unknown channel parameter is modeled as a stochastic process with known statistics. In particular, the phase noise is assumed modeled as a Wiener process, with incremental variance over a signaling interval equal to $\sigma_{\Delta}^{2}$. The FG corresponding to the joint a posteriori probability distribution of the information message bits given the received signal is built and the SPA is used to compute the posterior marginal distributions. Bit-by-bit decisions are then made, based on the resulting posterior marginals. The FG includes the knowledge of the unknown parameter statistics. Expectation over the unknown parameters is implicitly performed by the SPA as part of the marginalization.

We now briefly review the derivation of the low-complexity algorithm originally proposed in [9] that, in the following, will be denoted to as the "CBC" algorithm, from the names of the authors 4 . We will assume that the gain $A$ and the thermal noise variance, or equivalently the SNR, are perfectly known to the receiver. Then we will show how the algorithm can be extended in the presence of unknown gain and SNR. Let us focus on the above mentioned Wiener phase noise. While the SPA is well-suited to handle discrete random variables, characterized by a probability mass function (pmf), the channel parameters are, in this case, continuous random variables, characterized by

\footnotetext{
${ }^{4}$ The interesting reader can refer to [9] for the details of the derivation.
} 


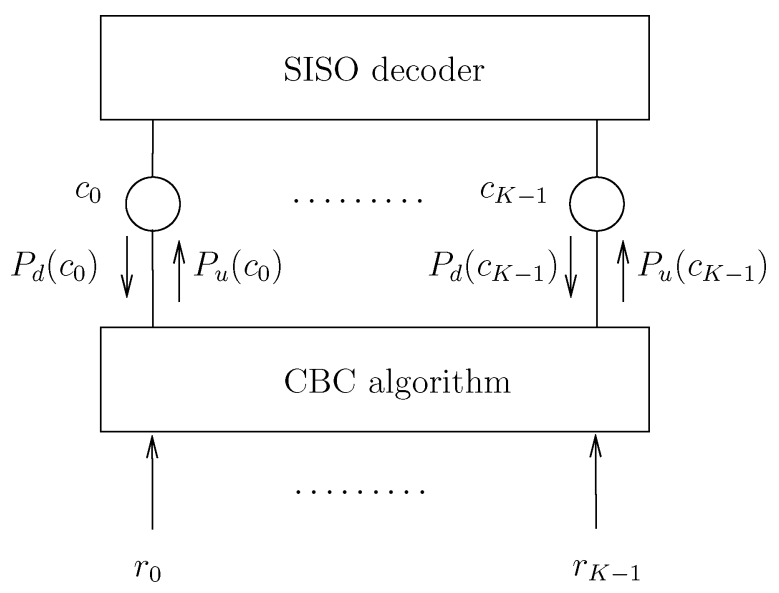

Fig. 3. Pictorial representation of the iterative receiver.

a pdf. The SPA for continuous random variables involves integration and computation of continuous pdfs, and it is not suited for direct implementation. A solution for this problem is suggested in [15] and consists of the use of canonical distributions, i.e., the pdfs computed by the SPA are constrained to be in a certain "canonical" family, characterized by some parameterization. Hence, the SPA has just to forward the parameters of the pdf rather than the pdf itself. Clearly, several different algorithms can be obtained depending on the choice of the canonical distribution.

In [9], an approach based on a Tikhonov parameterization has been proposed. It yields a one-dimensional forward-backward recursion that can be regarded (roughly speaking) as a non-linear version of the Kalman smoother. Remarkably, its performance is nearly as good as the discretized-phase approach (nearly optimal) with considerable lower complexity [9]. We can say that this algorithm has an unprecedented performance/ complexity tradeoff and for this reason it has been implemented in the context of the project "Joint Turbo Decoding and Synchronization," ESA Contract 18261/04/NL/AR, by Advantech, TurboConcept, and Eurecom. ${ }^{5}$

Let us consider the Fig. 3, which represents a pictorial description of the iterative receiver. Two blocks, namely the SISO decoder (e.g., the LDPC decoder in the DVB-S2 scenario) and the $\mathrm{CBC}$ algorithm, are iteratively activated and exchange themselves the extrinsic probabilities of the coded symbols. We denote by $P_{d}\left(c_{k}\right)$ the probability of the modulation symbol $c_{k}$ at time epoch $k$ provided by the decoder and by $P_{u}\left(c_{k}\right)$ the extrinsic a posteriori probability evaluated by the $\mathrm{CBC}$ algorithm. These probabilities are iteratively updated, but the explicit reference on the iteration number is dropped for simplicity.

The CBC algorithm is based on the following steps:

1) Given the messages $P_{d}\left(c_{k}\right), k=0,1, \ldots, K-1, c_{k} \in \mathcal{X}$, provided by the decoder, for $k=0,1, \ldots, K-1$, compute

$$
\alpha_{k}=A \sum_{c_{k}} P_{d}\left(c_{k}\right) c_{k}
$$

5The acronym "CBC" to denote the algorithm has appeared for the first time in the technical reports of this project. and

$$
\beta_{k}=A^{2} \sum_{c_{k}} P_{d}\left(c_{k}\right)\left|c_{k}\right|^{2}
$$

The complex parameter $\alpha_{k}$ and the real one $\beta_{k}$ are respectively the first and second order moments of the a priori pmf $P_{d}\left(c_{k}\right)$.

2) Forward recursion. It consists of the evaluation of a sequence of complex parameters, one for each time epoch, denoted to as $a_{f, k}$ and implicitly representing an estimate of the phase at time $k$. Let $a_{f, 0}=0$. For all $k=1,2, \ldots, K-1$, compute

$$
a_{f, k}^{\prime}=a_{f, k-1}+2 \frac{r_{k-1} \alpha_{k-1}^{*}}{2 \sigma^{2}+\beta_{k-1}-\left|\alpha_{k-1}\right|^{2}}
$$

and then

$$
a_{f, k}=\frac{a_{f, k}^{\prime}}{1+\sigma_{\Delta}^{2}\left|a_{f, k}^{\prime}\right|} .
$$

In the previous recursive equation, $\sigma_{\Delta}$ is, as already mentioned, the standard deviation of the increment $\Delta_{k}$ of the Wiener process. In the numerical results, we will consider a phase noise that cannot be modeled as a Wiener process. In that case, $\sigma_{\Delta}$ must be considered as a design parameter to be optimized by computer simulation for the phase noise at hand.

3) Backward recursion. Similarly to the forward recursion, a sequence of complex parameters $a_{b, k}$ is recursively updated during this step. Let $a_{b, K-1}=0$. For all $k=$ $K-2, \ldots, 1,0$, compute

$$
a_{b, k}^{\prime}=a_{b, k+1}+2 \frac{r_{k+1} \alpha_{k+1}^{*}}{2 \sigma^{2}+\beta_{k+1}-\left|\alpha_{k+1}\right|^{2}}
$$

and then

$$
a_{b, k}=\frac{a_{b, k}^{\prime}}{1+\sigma_{\Delta}^{2}\left|a_{b, k}^{\prime}\right|} .
$$

4) The messages sent to the decoder for a new iteration will be, for all $k=0,1, \ldots, K-1$

$$
\begin{aligned}
P_{u}\left(c_{k}\right) & \propto \exp \left\{-A^{2} \frac{\left|c_{k}\right|^{2}}{2 \sigma^{2}}\right\} \mathrm{I}_{0}\left(\left|a_{f, k}+a_{b, k}+A \frac{r_{k} c_{k}^{*}}{\sigma^{2}}\right|\right) \\
& \simeq \exp \left\{-A^{2} \frac{\left|c_{k}\right|^{2}}{2 \sigma^{2}}+\left|a_{f, k}+a_{b, k}+A \frac{r_{k} c_{k}^{*}}{\sigma^{2}}\right|\right\} .
\end{aligned}
$$

where $\mathrm{I}_{0}(\cdot)$ is the modified Bessel function of the first kind of order zero. Equation (10) stems from the fact that, for large enough argument, $\mathrm{I}_{0}(x) \simeq e^{x}$.

Hence, the algorithm is based on a forward-backward schedule performed over the whole codeword. This inherently serial schedule increases the latency. Indeed, while the LDPC decoding can be highly parallelized at the expense of an increased hardware complexity, thus increasing the actual throughput, a similar parallelization is not straightforward in the CBC algorithm, which may therefore become the bottleneck of the iterative detection and decoding receiver.

We now propose a way to implement the CBC with a higher degree of parallelism, with a (practically negligible) loss in 
terms of energy efficiency. The frame is logically partitioned into several overlapped blocks, such that each of them starts and ends with a pilot field. At every iteration, an instance of the CBC algorithm is run over each block independently, but using, as initial values for $a_{f, k_{1}}$ and $a_{b, k_{2}}\left(k_{1}\right.$ and $k_{2}$ being the first and last symbol of the considered block), the values of the previous iteration evaluated at the last and first symbol of the previous and next block respectively. In this way, the parallelism does not lead to completely independent phase tracking over different blocks, since during iterations phase estimates propagate quickly through the frame.

\section{A. Gain and SNR Estimation Algorithm}

It is important to remark that the $\mathrm{CBC}$ algorithm requires the exact knowledge of the gain and of the SNR, since in (3) -(9) the gain $A$ and the noise variance $\sigma^{2}$ explicitly appear. On the other hand, the estimation of gain and SNR must be performed on a per-frame basis, since in the ACM mode two consecutive frames might carry different MODCODs formats. The problem of gain and SNR estimation has been widely studied in the past (for example, see [16]-[18]). However the application of these classical techniques to the DVB-S2 scenario raise an important issue: the presence of a strong phase noise. We propose to embed a new iterative gain/SNR estimation algorithm in the $\mathrm{CBC}$, in such a way a new low-complexity estimation step is carried out at every iteration, with the aim of iteratively improving the estimates. We verified by computer simulations (as shown in Section V) that in the DVB-S2 scenario a DA estimation algorithm based on the known preamble only (or possibly on the distributed pilot symbols, if present) can reach the accuracy required to have only a minor loss with respect to the case of known gain and SNR.

To estimate in a coherent way these unknown parameters, the knowledge of the phase noise samples is required. We employ the implicit phase estimates produced by the $\mathrm{CBC}$ algorithm for this purpose. We already mentioned that the arguments of the complex parameters $a_{f, k}$ and $a_{b, k}$, evaluated during the forward and backward recursions represent an estimate of the channel phase at time epoch $k$, given the past and future observed samples, respectively 6 . Thus, the best estimate of the phase at time $k$ at the end of the $\ell$-th iteration turns out to be

$$
\hat{\theta}_{k}^{(\ell)}=\arg \left(a_{f, k}^{(\ell)}+a_{b, k}^{(\ell)}\right)
$$

where the superscript $\ell$ denotes the iteration. Let us therefore denote as $\mathcal{P}$ a set of time epochs, such that $\forall k \in \mathcal{P}, c_{k}$ is known. By assuming $\hat{\theta}_{k}^{(\ell)}=\theta_{k}$, that is, the true phase at time $k$ coincides with its estimate evaluated by the CBC, which is a realistic hypothesis especially after some iterations, and applying the joint maximum likelihood (ML) strategy to the estimation of the parameters $\left(A, \sigma^{2}\right)$, it turns out that the estimates of gain and noise variance at the $(\ell+1)$-th iteration become

$$
\hat{\sigma}^{2(\ell+1)}=\frac{1}{2 \operatorname{card}(\mathcal{P})} \sum_{n \in \mathcal{P}}\left|r_{n}-\hat{A}^{(\ell)} c_{n} e^{j \hat{\theta}_{n}^{(\ell)}}\right|^{2}
$$

${ }^{6}$ On the other hand, it can be shown that the magnitude of the complex parameters $a_{f, k}$ and $a_{b, k}$ is instead inversely proportional to the phase estimation variance. Hence, the larger the magnitude, the higher the reliability of the phase estimate.

$$
\hat{A}^{(\ell+1)}=\frac{1}{\operatorname{card}(\mathcal{P})} \sum_{n \in \mathcal{P}} \operatorname{Re}\left[r_{n} c_{n}^{*} e^{-j \hat{\theta}_{n}^{(\ell)}}\right] .
$$

As the iterations proceed, better phase estimates are produced by the $\mathrm{CBC}$, thus refining also the estimates of the gain and noise variance. This approach will be denoted as DA/PED, i.e., data aided, since it uses only the known symbols, and phase estimate directed, since it performs coherent estimation by using the phase estimates coming from the CBC. Clearly, an initial guess of the parameters is required in order for the algorithm to bootstrap. A natural choice is $\hat{A}^{(0)}=E\{A\}$, i.e., its expected value, and $\hat{\sigma}^{2(0)}$ corresponding to the lowest SNR value for which the considered communication system is below a given bit-error rate (BER), the so-called SNR threshold value.

As a final remark, we point out that, if necessary, a modification of the proposed algorithm may be developed to take also advantage from the unknown (code) symbols during the estimation, by using the preliminary decision from the LDPC decoder, thus obtaining a DA-DD/PED version, where DD stands for decision directed. Anyway, we will not pursue this solution since we verified that in the DVB-S2 scenario the estimates based only on the known preamble are always sufficiently accurate.

\section{NUMERICAL RESULtS}

In this Section, the performance of the proposed estimation and detection algorithms is assessed by computer simulations. Unless otherwise specified, the phase noise we consider is the DVB-S2 compliant ESA phase noise model for consumer-grade equipment at a signaling rate of 10 Mbaud [9], [10].

\section{A. Performance of the Frequency Estimation Algorithm}

In order to evaluate the performance of the proposed frequency estimation algorithm, three kinds of computer simulations are employed. In the first one, we evaluate the outlier probability of the first step of the algorithm, that is the coarse DA estimation, versus $E_{S} / N_{0}, E_{S}$ being the received signal energy per modulation symbol and $N_{0}$ the one-sided noise power spectral density. In this case, the outlier probability is defined in the following way: let us assume that we want to keep and select through the decoder a maximum of $2 n+1$ estimates after the fine DA step. Hence, the algorithm works only if the estimation error of the first step lies inside an interval of width $(2 n+1) /\left(L_{d}+L_{p}\right)$ centered around zero. On the contrary, when the true frequency offset value does not belong to the set of estimates produced by the first two steps, the frame will be decoded incorrectly. Thus, in order to carry out correct decoding, the outlier probability must be lower than the target FER. In Fig. 4, the outlier probability for different values of the maximum number of employed estimates is shown. As it can be seen, depending on the working SNR, a different number of estimates has to be kept and fed to the selection step. For example, for higher-order modulation formats, characterized by a large working SNR, 3 trial values are sufficient whereas, in all other cases, we need that the coarse + fine DA steps produce 5 estimates. The same figure also tells that with the standardized pilot distribution a classical DA frequency estimation strategy, producing only one estimate, fails. 


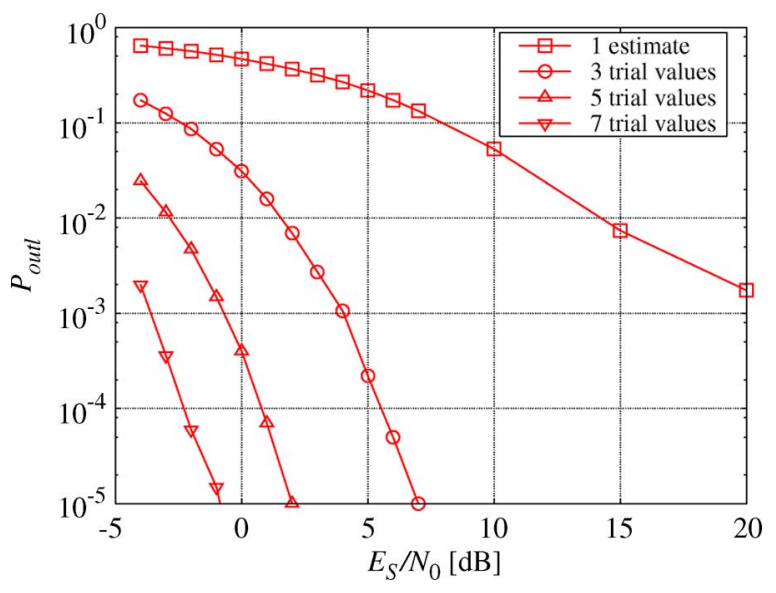

Fig. 4. Outlier probability of the coarse DA step for different values of the number of estimated values fed to the selection step.

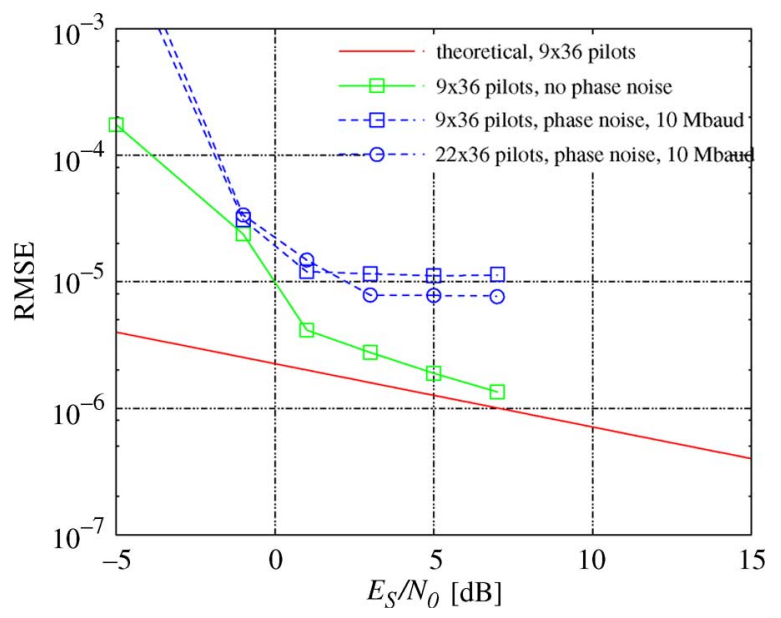

Fig. 5. Performance of the code-aided DA estimation algorithm in case of selection of the correct frequency estimate.

In the second computer simulation we evaluate the estimation accuracy of the first two steps under the hypothesis of genie-selection, by taking into account a total of five estimated values. In other words, we consider the estimate $\hat{\nu}_{1}+\hat{\nu}_{2}+\left(\hat{\ell} /\left(\left(L_{d}+\right.\right.\right.$ $\left.\left.L_{p}\right) T\right)$ ) being $\hat{\ell}$ (which can take on the values $0, \pm 1, \pm 2$ ) the integer ensuring the lowest error. Fig. 5 refers to the estimation accuracy of the proposed algorithm, under the hypothesis of correct selection. As it can be seen, in the absence of phase noise the estimation accuracy leans on the following theoretical RMSE curve, valid for large SNR:

$$
R M S E_{\text {theor }}=\sqrt{\frac{2 P-3}{2 \pi^{2} L_{p}\left(L_{d}+L_{p}\right)^{2}(P-1)^{2} \frac{E_{S}}{N_{0}} T^{2}}} .
$$

Equation (14) was obtained by substituting $z_{k}=r_{k} c_{k}^{*}$ in (2), neglecting the noise by noise terms and replacing $\arg (1+u)$ with $\operatorname{Im}(u)$, which is a valid approximation for small $u$. On the other hand, it is known that, in the presence of phase noise, a floor in the RMSE of the frequency estimator appears, regardless of the employed estimation algorithm, namely it is not possible to reduce the RMSE below a given threshold by simply increasing the SNR [2]. In addition, we can observe that the performance is practically independent of the number of considered pilots

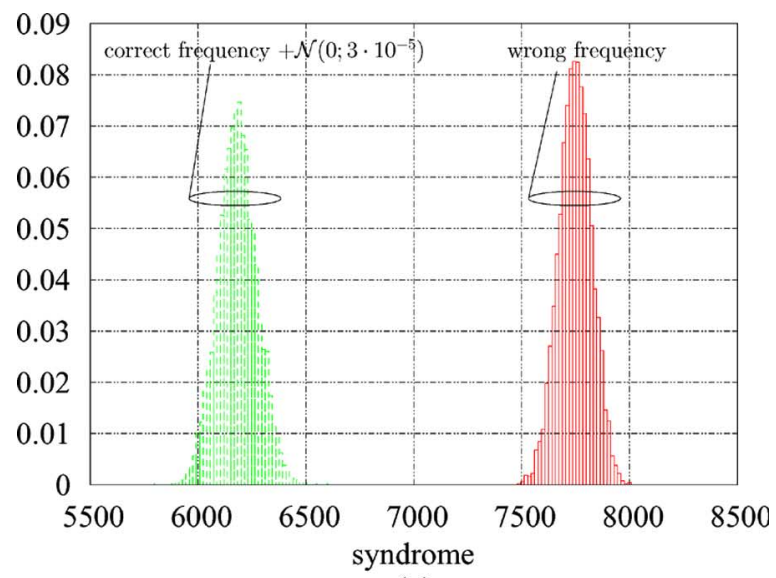

(a)

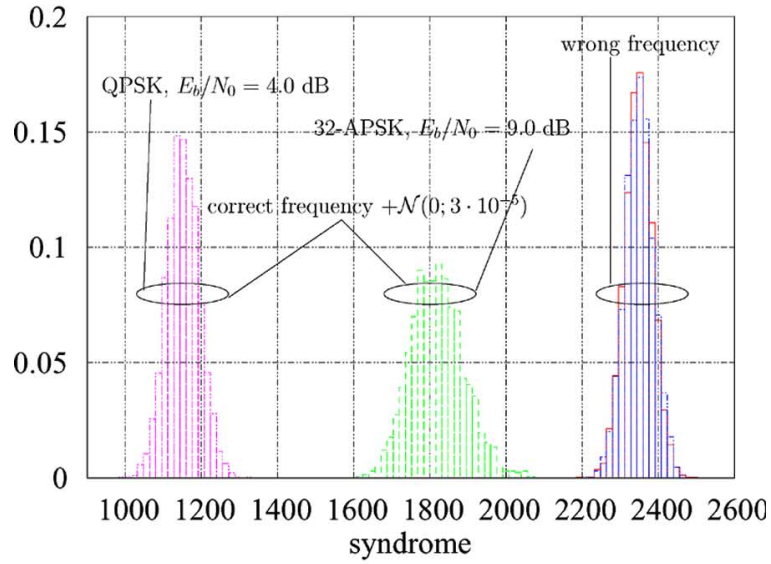

(b)

Fig. 6. Syndrome histogram at the end of the first iteration. (a) LDPC code with $r=2 / 3,8$-PSK modulation and $E_{b} / N_{0}=3.5 \mathrm{~dB}$; (b) LDPC code with $r=9 / 10$, QPSK modulation at $E_{b} / N_{0}=4.0 \mathrm{~dB}$ and 32-APSK modulation at $E_{b} / N_{0}=9.0 \mathrm{~dB}$

fields (22 in the case of QPSK or 9 in the case of 32-APSK, considering also the last symbols of the preamble as a pilot field). When only five estimates are employed, the target accuracy that let the algorithm for joint detection and decoding described in Section IV work, is reached for $E_{S} / N_{0} \geq 1 \mathrm{~dB}$. To obtain a better performance, seven estimates are necessary.

Finally, in the last computer simulation, we evaluate the probability that the code-aided selection algorithm chooses the wrong estimate. Clearly, when this event occurs the LDPC decoder produces a very large number of bit errors. Hence, we must ensure that the probability of this event is sufficiently lower than the target FER. In Fig. 6(a), the histogram of the code syndrome for the 8-PSK modulation, with the low-density parity-check (LDPC) of rate $r=2 / 3$ at $E_{b} / N_{0}=3.5 \mathrm{~dB}, E_{b}$ being the received signal energy per information bit, is shown. The leftmost histogram was obtained by considering a residual normalized frequency error with a Gaussian distribution having zero mean and a standard deviation $3 \cdot 10^{-5}$, independently generated frame by frame, while the rightmost histogram refers to wrong frequency values [namely, with absolute errors larger than $\left.1 /\left(2\left(L_{d}+L_{p}\right)\right)\right]$. The detection is performed by means of the CBC algorithm described in Section IV. Only one iteration of the detector and decoder is allowed. As it can be seen, there is a huge separation between the histograms. Hence the 
proposed technique is viable for the considered scenario: after one iteration of detector and decoder, if the code syndrome is below a threshold of 7000 , we can say that we have found the right frequency estimate. Otherwise, we go ahead to the next value. Clearly, this separation increases for larger SNR values.

Since, in a code-aided technique, the most critical case occurs for the less robust code, in Fig. 6(b) we consider the LDPC code with $r=9 / 10$, along with the two farthest modulations: QPSK and 32-APSK at $E_{b} / N_{0}=4.0 \mathrm{~dB}$ and $E_{b} / N_{0}=9.0 \mathrm{~dB}$, respectively. In the case of wrong frequencies, the syndrome histograms for the two different modulations are more or less overlapped. On the contrary, the separation between the histograms for correct and wrong frequency estimates are completely different for the two modulations. The most critical case occurs for 32-APSK, due to its lower robustness to phase errors, but still in this case there is a separation high enough to ensure that, by using a syndrome threshold value of about 2150 , the event "choice of a wrong frequency estimate" has a negligible probability.

\section{B. Performance of the CBC Algorithm}

The performance of the proposed detection algorithm with the proposed hybrid schedule is assessed by computer simulations in terms of BER versus $E_{b} / N_{0}$. Excepting the iterations necessary to select the frequency estimate within the set of $2 n+1$ trial values 7 , a maximum of 50 iterations of the iterative receiver is allowed. For each simulated point, a minimum of 50 frame errors is counted.

In all simulated cases, pilot symbols, following the standardized distribution, are inserted in the transmitted codeword in order to make the iterative decoding algorithm bootstrap. Pilot symbols involve a slight decrease in the effective information rate, resulting in an increase in the required signal-to-noise ratio. This increase has been introduced artificially in the curve labeled "known phase" for the sake of comparison. Hence, the gap between the "known phase" curve and the others is not related to the rate decrease due to pilot symbols.

Despite the CBC algorithm was developed with a Wiener phase noise model in mind [9], in the computer simulations we consider the DVB-S2 compliant ESA phase noise model [9], [10]. In Fig. 7(a), we consider four standardized LDPC codes with codewords of length 64800 [1], namely a rate-1/2 code mapped onto a QPSK modulation, a rate-2/3 code mapped onto an 8-PSK modulation, a rate-3/4 code mapped onto a 16-APSK modulation and a rate- $4 / 5$ code mapped onto a 32 -APSK modulation. The above mentioned phase noise ESA model is considered, for a signaling rate of $10 \mathrm{Mbaud}$ or $25 \mathrm{Mbaud}$, and the gain and the noise variance are known at the receiver. The CBC algorithm exhibits only a minor loss due to phase noise, less than $0.1 \mathrm{~dB}$ for QPSK, 8-PSK and 16-APSK modulations. ${ }^{8}$ On the contrary, the loss for 32-APSK is larger, due to the larger sensitivity of this constellation to phase mismatches as well as to the

\footnotetext{
${ }^{7}$ Since we may stop the selection procedure when the code syndrome falls below the threshold, on average $n$ more iterations are necessary for the selection step.

${ }^{8}$ In [10] a PLL-based synchronization algorithm was proposed and its performance investigated in the DVB-S2 scenario. It turns out that, despite the (limited) complexity savings of this algorithm with respect to the proposed $\mathrm{CBC}$, its performance is not acceptable because in many of the proposed MODCOD the relevant BER curve exhibits a slope different to the coherent case and it is not able to reach the desired BER.
}

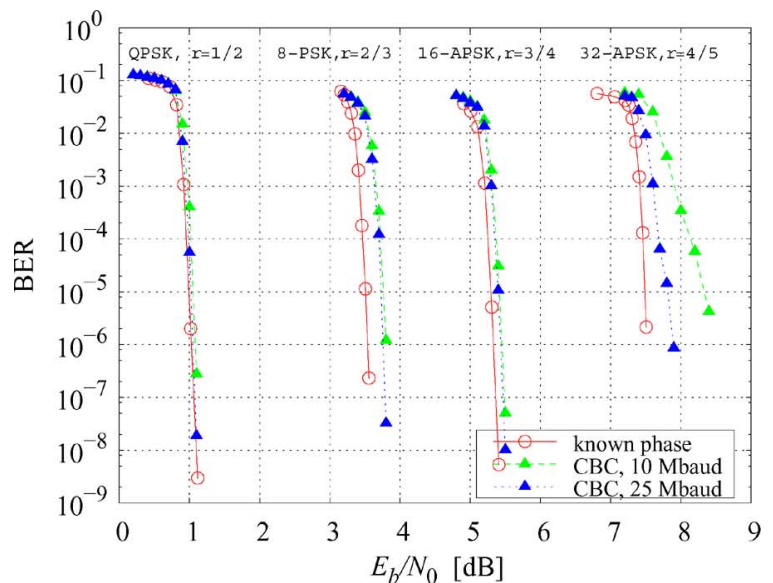

(a)

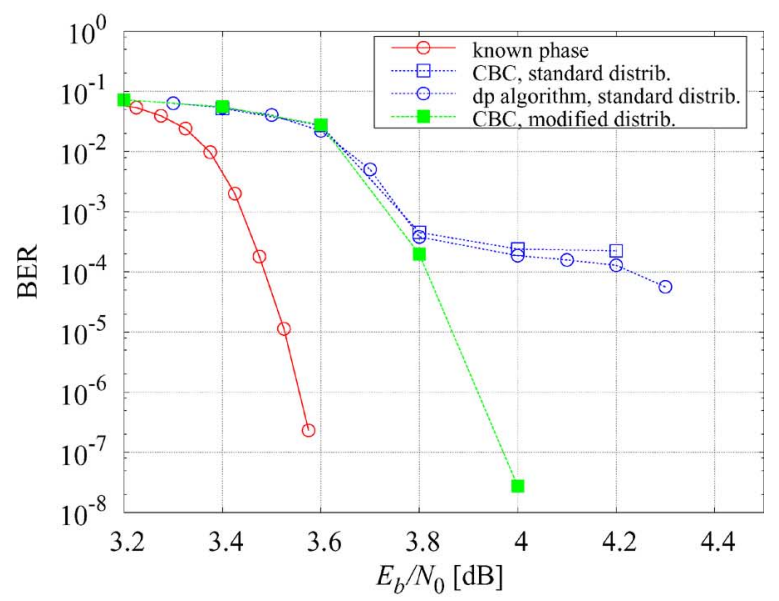

(b)

Fig. 7. Performance of the proposed algorithm based on Tikhonov param eterization. The ESA phase model for several signaling rates is considered. (a) QPSK, 8-PSK, 16-APSK, and 32-APSK modulations, with standard pilot distribution; (b) LDPC code with $r=2 / 3$ mapped onto the 8-PSK modulation at a signaling rate of 1 Mbaud.

higher code rate. Moreover, as it can be seen, the loss is larger for lower signaling rates, since the lower the signaling rate the faster the phase noise.

In Fig. 7(b), we consider the rate-2/3 code mapped onto an 8-PSK, but the DVB-S2 compliant phase noise for a signaling rate of $1 \mathrm{Mbaud}$ is employed. Two pilot symbol distributions are taken into account. "Standard" refers to the standardized distribution (i.e., 36 pilot symbols every 1476 transmitted symbols [1]) while "modified" refers to a more sparse distribution characterized by 3 pilot symbols every 123 transmitted symbols. It is worth noticing that, despite the insertion rate is the same for the two cases, the performance of the CBC algorithm for the modified distribution is much better. The bad performance of the CBC algorithm for the standard distribution in the considered scenario is due to the fact that the phase noise corresponding to $1 \mathrm{Mbaud}$ varies faster than that corresponding to $10 \mathrm{Mbaud}$, thus leads to a non-negligible loss if the pilot fields are too far one of each other, as in the standard pilot distribution. This is confirmed by the performance of the practically optimal algorithm based on phase discretization (dp algorithm in the figure) [9] which is comparable to that of the CBC algorithm. 


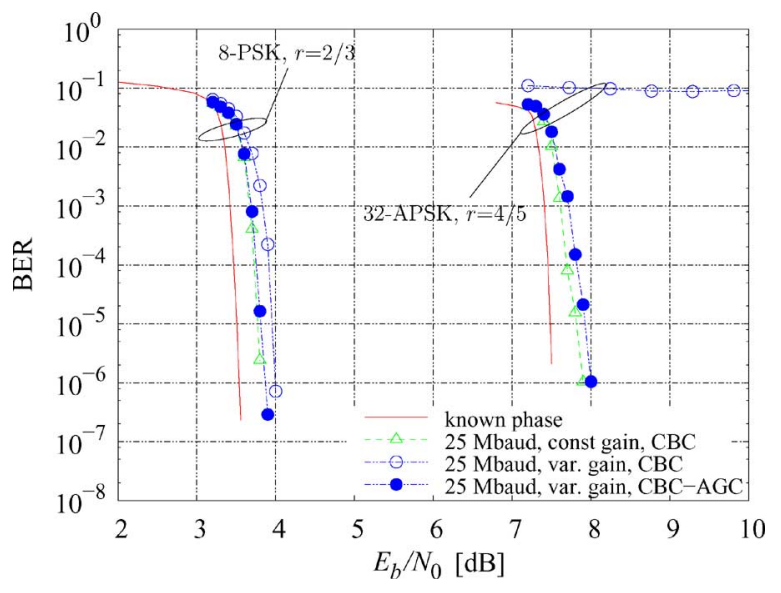

Fig. 8. Performance of the proposed algorithm. The ESA phase model for a signaling rate of 25 Mbaud is considered along with 8-PSK and 32-APSK modulations.

The performance of the proposed gain and SNR estimation algorithm embedded in the CBC for 8-PSK and 32-APSK is shown in Fig. 8. Along with the known-phase/known-gain curve, three other scenarios are considered: i) known gain and SNR, presence of phase noise and the classical CBC algorithm; ii) unknown random gain, unknown SNR, presence of phase noise and the classical $\mathrm{CBC}$ algorithm; iii) unknown random gain, unknown SNR, presence of phase noise and the CBC algorithm with embedded gain and SNR estimation (based only on the preamble of 90 symbols).

Some observations can be drawn from Fig. 8. In the presence of a random unknown gain and an unknown SNR, the loss of the $\mathrm{CBC}$ algorithm increases due to the fact that no explicit estimation and compensation if performed. It is particularly worth the result for 32-APSK, for which the receiver without the explicit estimation cannot reach BER values less than $10^{-1}$. This is well in line with the fact that APSK modulations are much more sensitive to gain and SNR mismatches. Finally, by considering the proposed gain and SNR estimators along with the CBC algorithm, the performance practically coincides with the case of known gain and SNR. Therefore the proposed algorithm is very effective in estimating and compensating gain and SNR.

\section{CONCLUSIONS}

We discussed pilot-symbol-assisted carrier synchronization in future DVB-S2 receivers. A single low-complexity and robust solution has been identified and its performance analysed. In the absence of pilots, however, this solution cannot be employed, since the fine frequency estimator heavily exploits pilots and in addition, the sensitivity of the $\mathrm{CBC}$ algorithm to frequency errors highly increases in the absence of pilots. On the other hand, to our best knowledge, for the considered strong phase noise there is not a single solution that works for all modulation and coding formats and all signaling rates and for some cases the carrier synchronization is still an open problem.

We would like to remark that a more sparse pilot distribution would have been beneficial to reduce the receiver com- plexity and improve its performance. In fact, from a computational complexity point of view the frequency estimation algorithm would have been simplified since the selection step could have been avoided. From a performance point of view, the detection algorithm would have been able to cope with the strongest phase noise, namely that for the lowest signaling rate.

\section{REFERENCES}

[1] "ETSI - DVBS2 74r13, Digital Video Broadcasting (DVB): Second generation framing structure, channel coding and modulation systems for Broadcasting, Interactive Services, News Gathering and other broadband satellite applications," 2003.

[2] A. Barbieri, D. Bolletta, and G. Colavolpe, "On the Cramer-Rao bound for carrier frequency estimation in the presence of phase noise," in Proc. IEEE Global Telecommun. Conf., St. Louis, MO, U.S.A., 2005, pp. $720-724$.

[3] A. Barbieri, A. Cero, D. Fertonani, and G. Colavolpe, BSDT-Technical note TN01: Modem algorithms design specification for the broadband forward link with linear modulations-Part 1: Carrier synchronization ESA Contract No. 19370, July 2006, Tech. Rep., available upon request.

[4] U. Mengali and A. N. D'Andrea, Synchronization Techniques for Digital Receivers (Applications of Communications Theory). New York: Plenum Press, 1997.

[5] D. Fertonani, A. Barbieri, G. Colavolpe, and D. Delaruelle, "Estimation and compensation of linear amplitude distortions," in 9-th Intern. Work. on Signal Processing for Space Commun., Noordwijk, The Netherlands, Sept. 2006.

[6] G. Albertazzi, S. Cioni, G. E. Corazza, M. Neri, R. Pedone, P. Salmi, A. Vanelli-Coralli, and M. Villanti, "On the adaptive DVB-S2 physical layer: design and performance," IEEE Wireless Communications, vol. 12, pp. 62-68, Dec. 2005.

[7] K. A. Barratt, P. Coulton, and B. Honary, "Defining performance limits for turbo-code assisted synchronization in DVB-S systems," IEEE Trans. Broadcasting, vol. 47, pp. 348-356, Dec. 2001.

[8] U. Mengali and M. Morelli, "Data-aided frequency estimation for burst digital transmission," IEEE Trans. Commun., vol. 45, pp. 23-25, Jan. 1997.

[9] G. Colavolpe, A. Barbieri, and G. Caire, "Algorithms for iterative decoding in the presence of strong phase noise," IEEE J. Select. Areas Commun., vol. 23, no. 9, pp. 1748-1757, Sept. 2005.

[10] A. Ginesi, D. Fittipaldi, A. Bigi, and R. De Gaudenzi, Pilot-aided carrier synchronization techniques for broadband satellite transmissions, Sept. 2003, ESA-ESTEC, Tech. Rep.

[11] D. C. Rife and R. R. Boorstyn, "Single tone parameter estimation from discrete-time observations," IEEE Trans. Inform. Theory, vol. 20, pp. 591-598, September 1974.

[12] J. A. Gansman, J. V. Krogmeier, and M. P. Fitz, "Single frequency estimation with non-uniform sampling," in Proc. Asilomar Conf. Signals, Systems, Comp., Nov. 1997, pp. 399-403.

[13] I. Motedayen-Aval and A. Anastasopoulos, "Polynomial-complexity noncoherent symbol-by-symbol detection with application to adaptive iterative decoding of turbo-like codes," IEEE Trans. Commun., vol. 51, pp. 197-207, Feb. 2003.

[14] C.-H. Hsu and A. Anastasopoulos, "Design and analysis of joint data detection and frequency/phase estimation algorithms," IEEE J. Select. Areas Commun., vol. 23, no. 9, pp. 1707-1717, Sept. 2005.

[15] A. P. Worthen and W. E. Stark, "Unified design of iterative receivers using factor graphs," IEEE Trans. Inform. Theory, vol. 47, no. 2, pp. 843-849, Feb. 2001

[16] D. R. Pauluzzi and N. C. Beaulieu, "A comparison of SNR estimation techniques for the AWGN channel," IEEE Trans. Commun., vol. 48, pp. 1681-1691, Oct. 2000.

[17] T. A. Summers and S. G. Wilson, "SNR mismatch and online estimation in turbo decoding," IEEE Trans. Commun., vol. 46, no. 4, pp. 421-423, Apr. 1998.

[18] A. Wiesel, J. Goldberg, and H. Messer, "Non-data-aided signal-tonoise-ratio estimation," in Proc. IEEE Intern. Conf. Commun., April/May 2002, pp. 197-201. 